\title{
Theoretical Studies on the Longitudinal Inhomogeneity of Track Stiffness and a Track Status Estimation Method
}

\author{
Wen Bai ${ }^{1}$ and Lei $\mathrm{Xu} \mathbb{1}^{2,3}$ \\ ${ }^{1}$ Key Laboratory of Earthquake Engineering and Vibration Engineering, Institute of Engineering Mechanics, \\ China Earthquake Administration, Harbin, Heilongjiang 150080, China \\ ${ }^{2}$ School of Civil Engineering, Central South University, Changsha 410075, China \\ ${ }^{3}$ State Key Laboratory of Traction Power, Southwest Jiaotong University, Chengdu 610031, China \\ Correspondence should be addressed to Lei Xu; xu.lei@csu.edu.cn
}

Received 22 August 2020; Revised 29 March 2021; Accepted 1 April 2021; Published 21 April 2021

Academic Editor: Timothy O. Olawumi

Copyright (C) 2021 Wen Bai and Lei Xu. This is an open access article distributed under the Creative Commons Attribution License, which permits unrestricted use, distribution, and reproduction in any medium, provided the original work is properly cited.

\begin{abstract}
Due to material multiplicity, rigid-flexible hybrid and environmental variations, and so forth, the properties of the tracks will be inevitably changed due to the cyclic loads temporally and spatially. In this paper, a theoretical study is conducted to clarify the influence of the longitudinal inhomogeneity of track stiffness on system responses and then a general state estimation method is proposed to inversely predict the parametric distribution of the tracks. To achieve these purposes, a 3D nonlinear vehicle-track coupled model is firstly constructed, where track systems are established by the finite element method with random parameters. Based on this dynamic model, the distribution characteristics of system responses due to random parametric excitations are properly revealed. The numerical studies show that the longitudinal inhomogeneity of track supporting stiffness has significant effects on system responses; besides, the response distributions are varied against different parametric distributions. Finally, a representative example is demonstrated to show the effectiveness of the proposed track state estimation method.
\end{abstract}

\section{Introduction}

Railway tracks serve as an essential element in supporting the running vehicles and guiding their dynamical motions. Compared to vehicles, the railway tracks are characterized by the complexity in material multiplicity, rigid-flex hybrid, longitudinal large scales, environmental changes, and so forth. Apart from the construction deviations, the track status, generally regarded as the track geometric such as track irregularities, and parametric status, such as the stiffness softening, are constantly affected by the cyclic vehicle loads and are nonuniformly and randomly evolved against the railway operations.

In most of the vehicle-track dynamic simulations [1-8], track irregularities at time or frequency domain are treated as the main system excitations, and the vehicle and the tracks are considered as steady and constant systems. The longitudinal inhomogeneity and randomness of the tracks and their dynamic influence are neglected. In the field of vehicle-track dynamics nowadays, irrespective of it being in local-micro or in global-macro, it seems to be a growing trend to meticulously take into account the parametric excitation in a way approaching the essences. Generally, the parametric excitation is originated from the inhomogeneity of system properties, especially with uncertainties.

In previous researches, the cognitions of the track parameter unevenness are mainly characterized from frequency domain. Wu and Thompson [9] investigated the wheel/rail interactions and system responses considering the spatially varying stiffness of a ballasted track using a quasi-static method. In their method, the time-domain model is equivalently transformed from the space-varying receptance based on the linearity of the tracks and wheelrail interactions. Nélain and Vincent [10] carried out a deep research into the influence of sleeper periodicity, randomness and variance of the sleeper stiffness, and the 
foundation stiffness along the track on the parametric unevenness. Additionally, Verachtert et al. [11] investigated the relation between the unevenness of slab or track bed and the perceived unevenness derived by a train in frequency domain.

Specific to systematic dynamics, Mao et al. [12] conducted an interesting work in random dynamic analysis of a train-bridge system, where the random system parameters are considered, while the longitudinal inhomogeneity of the system is not involved; similar work can also be found in [13, 14]. Xu and Zhai [15] made an extensive research on vehicle-track stochastic dynamics, in which the randomicity of the vehicle-track system in excitations and dynamic parameters is wholly considered. Besides, the full view of track irregularities is depicted in matrix forms to construct a highly efficient probabilistic model. From the perspective of microanalysis, Corrêa et al. [16] regarded the granular medium as a stochastic heterogeneous continuum medium and calibrated a heterogeneous continuum model to model the railway ballast samples.

However, a universal method where the nonlinear wheel-rail contacts and the random track system with parametric unevenness along the track subject to a vehicle loading are not fully considered for studies in three-dimensional (3D) space. Besides revealing the dynamic effects of track inhomogeneity in vehicle-track dynamics, another challenge that derives from the influenc of track inhomogeneity on system response is how to estimate the track parametric status from the responses conversely. Although it might be possible to experimentally detect the track parameters or support stiffness within a small track section, it is difficult to measure the parametric state at large scales.

$\mathrm{Au}$ et al. [17] described a method for the vehicle parameters subjected to multispan continuous bridges using genetic algorithm, where the vehicle is modelled as a 2degree-of-freedom system. Similar work can be consulted in $[18,19]$. These approaches are practical rather than simplified systems only. Recently, Onat et al. [20] proposed a method to estimate the maximum friction coefficient in wheel-rail interfaces based on a sigma point Kalman-filterbased scheme. Up to now, there have been limited reports in literature which provide some theoretical guidance in the estimation of track parametric status, especially the parameters randomly distributed along a large length of the tracks.

In this paper, we pay special attention to clarifying the influence of track parametric inhomogeneity in a spatially longitudinal direction on system responses. Based on the clarification of the dynamic effects of track longitudinal inhomogeneity, a further research on inverse approach of estimating the track parametric status will be proposed. The random theories have indicated that only statistical information is required to construct a random model; thus, the estimation of the track parameters is concentrated on estimating the parametric distributions.
Hence, instead of the experimental researches and validations, the work presented is mainly conducted from a theoretical view using numerical methods.

\section{Track Longitudinal Inhomogeneity and Its Coupled Dynamics with the Vehicle}

Figure 1 shows two commonly used track types: ballasted track and ballastless track.

In this paper, the railway tracks are modelled as a ballastless system with rails, track slabs, and a winkle foundation. The rails and the track slabs are connected by discrete rail pads, and the cement asphalt (CA) mortar is applied to continuously provide elastic supports. Both the rail pads and CA mortar are treated as linear spring-dashpot elements.

In the track slab system, the concrete track slabs including the rails are essentially continuum medium in longitudinal direction. Therefore, we will meticulously construct their dynamic matrices using finite element method (FEM).

2.1. Matrix Construction for the Tracks with Inhomogeneity. Here a "Lateral Finite Strip and Slab Segment" method [21] is applied to describe the shape function of the track slab. For arbitrary point $o$ within the track slab element (TSE), as shown in Figure 2, its vertical displacement can be expressed as

$$
W_{o}=\mathbf{N}_{\mathrm{V}} \delta_{i j}
$$

with

$$
\begin{aligned}
\mathbf{N}_{V o}= & {\left[1-3\left(\frac{s_{y o}}{l}\right)^{2}+2\left(\frac{s_{y o}}{l}\right)^{3}-\left(\frac{s_{y o}}{l}-2\left(\frac{s_{y o}}{l}\right)^{2}+\left(\frac{s_{y o}}{l}\right)^{3}\right) l 3\left(\frac{s_{y o}}{l}\right)^{2}\right.} \\
& \left.-2\left(\frac{s_{y o}}{l}\right)^{3}\left(\left(\frac{s_{y o}}{l}\right)^{3}-\left(\frac{s_{y o}}{l}\right)^{2}\right) l\right], \\
\delta_{i j}= & {\left[W_{i}, \theta_{X i}, W_{j}, \theta_{Y j}\right]^{T}, }
\end{aligned}
$$

in which $W_{i}$ and $\theta_{X i}$ denote vertical displacement and angular displacement around $X$-axis for point $i$ and $W_{j}$ and $\theta_{X j}$ denote vertical displacement and angular displacement for point $j ; s_{y o}$ is the local $Y$-coordinate of point $o$; $l$ is the length of TSE along $Y$-axis.

Meanwhile, the vertical displacement and angular displacement in $\delta_{i j}$ can be further expressed as

$$
\begin{aligned}
W_{i} & =\mathbf{N}_{V i}\left[W_{a}, \theta_{Y a}, W_{b}, \theta_{Y b}\right]^{T}, \\
\theta_{X i} & =\mathbf{N}_{T i}\left[\theta_{X a}, \theta_{X b}\right]^{T}, \\
W_{j} & =\mathbf{N}_{V j}\left[W_{c}, \theta_{Y c}, W_{d}, \theta_{Y d}\right]^{T}, \\
\theta_{X j} & =\mathbf{N}_{T j}\left[\theta_{X c}, \theta_{X d}\right]^{T},
\end{aligned}
$$

with 


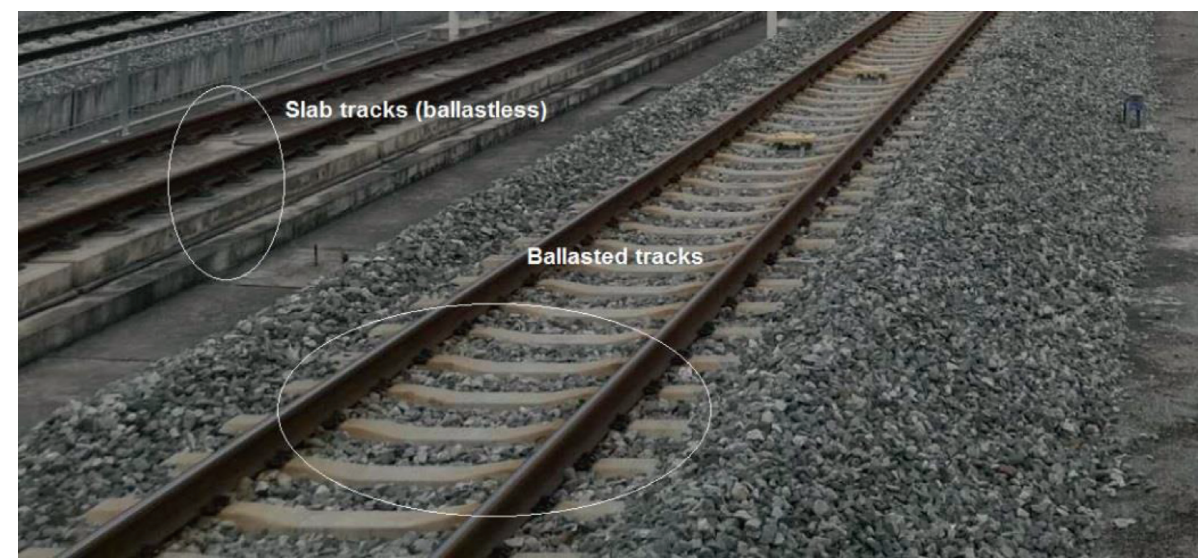

FIGURE 1: Typical track systems.

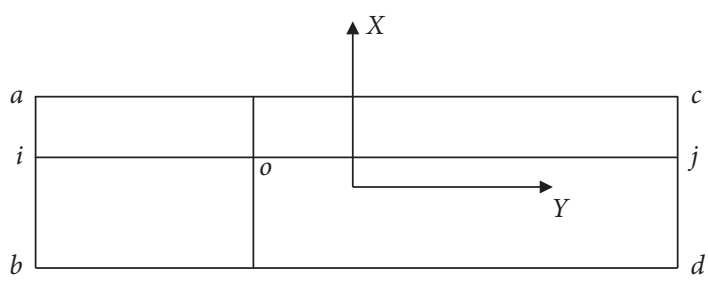

FIgURE 2: Top view of the TSE.

$$
\begin{aligned}
& \mathbf{N}_{V i}=\left[1-3\left(\frac{s_{x i}}{w}\right)^{2}+2\left(\frac{s_{x i}}{w}\right)^{3}-\left(\left(\frac{s_{x i}}{w}\right)-2\left(\frac{s_{x i}}{w}\right)^{2}+\left(\frac{s_{x i}}{w}\right)^{3}\right) w 3\left(\frac{s_{x i}}{w}\right)^{2}-2\left(\frac{s_{x i}}{w}\right)^{3}\left(\left(\frac{s_{x i}}{w}\right)^{3}-\left(\frac{s_{x i}}{w}\right)^{2}\right) w\right] \\
& \mathbf{N}_{T i}=\left[1-\left(\frac{s_{x i}}{w}\right)\left(\frac{s_{x i}}{w}\right)\right], \\
& \mathbf{N}_{V j}=\left[1-3\left(\frac{s_{x j}}{w}\right)^{2}+2\left(\frac{s_{x j}}{w}\right)^{3}-\left(\left(\frac{s_{x j}}{w}\right)-2\left(\frac{s_{x j}}{w}\right)^{2}+\left(\frac{s_{x j}}{w}\right)^{3}\right) w 3\left(\frac{s_{x j}}{w}\right)^{2}-2\left(\frac{s_{x j}}{w}\right)^{3}\left(\left(\frac{s_{x j}}{w}\right)^{3}-\left(\frac{s_{x j}}{w}\right)^{2}\right) w\right], \\
& \mathbf{N}_{T j}=\left[1-\left(\frac{s_{x j}}{w}\right)\left(\frac{s_{x j}}{w}\right)\right],
\end{aligned}
$$

in which $s_{x i}$ and $s_{x j}$ are, respectively, the local $X$-coordinate for points $i$ and $j ; w$ is the width of TSE along $X$-coordinate. $\theta_{Y}$ is the angular displacement around $Y$-axis.

Accordingly, by combining equations (1) and (3), the interpolation function for vertical displacement of $o$ can be written by

$$
\mathbf{N}_{\mathrm{v}, o}\left(s_{x o}, s_{y o}\right)=\left[\mathbf{N}_{V}(1) \mathbf{N}_{V}, \mathbf{N}_{V}(2) \mathbf{N}_{T}, \mathbf{N}_{V}(3) \mathbf{N}_{V}, \mathbf{N}_{V}(4) \mathbf{N}_{\mathrm{T}}\right]_{s_{x i}=s_{x j}=s_{x o}} .
$$

It is assumed that the dynamical parameter, for example, vertical stiffness of the cement asphalt mortar, follows a function $v=f_{v}(x, y)$. Obviously, the stiffness matrix induced by the vertically elastic interactions between the track slabs and the subgrade can be calculated:

$$
\mathbf{K}_{\mathbf{v}}^{\mathrm{e}}=f_{\mathbf{v}}\left(s_{x o}, s_{y o}\right) \int_{-(w / 2)}^{(w / 2)} \int_{-(l / 2)}^{(l / 2)} \mathbf{N}_{\mathrm{v}, o}^{\mathrm{T}} \mathbf{N}_{\mathrm{v}, o} \mathrm{~d} s_{y o} \mathrm{~d} s_{x o} .
$$

Certainly, the matrix induced by the elastic deformation energy of the thin-plate track slab element can be obtained by

$$
\mathbf{K}_{s}^{e}=\int_{-(l / 2)}^{(l / 2)} \int_{-(w / 2)}^{(w / 2)} \mathbf{B}^{\mathrm{T}} \mathbf{D} \mathbf{B} \mathrm{d} s_{x o} \mathrm{~d} s_{y o}
$$

with 


$$
\begin{aligned}
\mathbf{B}= & -\left[\frac{\partial^{2} \mathbf{N}_{v, o}}{\partial s_{y o}^{2}} \frac{\partial^{2} \mathbf{N}_{v, o}}{\partial s_{x o}^{2}} \frac{\partial^{2} \mathbf{N}_{v, o}}{\partial s_{y o} \partial s_{x o}}\right]^{T}, \\
\mathbf{D}= & \frac{E_{s}\left(s_{x o}, s_{y o}\right) H_{s}\left(s_{x o}, s_{y o}\right)^{3}}{12\left(1-\mu\left(s_{x o}, s_{y o}\right)^{2}\right)} \\
& \cdot\left[\begin{array}{cc}
1 & \mu\left(s_{x o}, s_{y o}\right) \\
\mu\left(s_{x o}, s_{y o}\right) & 0 \\
1 & 0 \\
0 & \frac{\left(1-\mu\left(s_{x o}, s_{y o}\right)\right)}{2}
\end{array}\right],
\end{aligned}
$$

in which $E_{\mathrm{s}}$ denotes Young's modulus of the track slab; $\mu$ denotes Poisson's ratio; $H_{\mathrm{s}}$ denotes the height of the track slab.

To other matrices of the track components (e.g., the rails) with continuum materials, the same manner as above can be applied, in which one needs to replace the specific parameters with spatially varied function (discrete).

The detailed expressions have been listed in [6]; no more illustrations are therefore given for brevity.

Two other issues for the construction and computation of the random track system in large length will be encountered:

(i) Computational consumption for large matrices

(ii) Boundary condition in long-length calculation

For solving the above matters, a cyclic calculation method presented in $[22,23]$ will be applied in the computations.

2.2. Construction of Coupled Dynamics with the Vehicle. Following the work presented in [1], the track vibration accompanied by its coupling effects to a moving vehicle or train can be depicted mechanically and physically, as shown in Figure 3. In this work, the vehicle is considered as a multi-rigid-body system, including one body, two bogie frames, and four wheelsets. Each body has five degrees of freedom, that is, bounce $Z_{V}$, transverse $Y_{V}$, roll $\theta_{V}$, yaw $\psi_{V}$, and pitch $\beta_{V}$.

Without loss of generality, the dynamic equations of motion for the vehicle-track interactions can be expressed by

$$
\begin{aligned}
& {\left[\begin{array}{cc}
\mathbf{M}_{V V} & 0 \\
0 & \mathbf{M}_{T T}
\end{array}\right]\left\{\ddot{\mathbf{X}}_{V} \ddot{\mathbf{X}}_{T}\right\}+\left[\begin{array}{cc}
\mathbf{C}_{V V} & 0 \\
0 & \mathbf{C}_{T T}
\end{array}\right]\left\{\begin{array}{c}
\dot{\mathbf{X}}_{V} \\
\dot{\mathbf{X}}_{T}
\end{array}\right\}} \\
& +\left[\begin{array}{cc}
\mathbf{K}_{V V} & 0 \\
0 & \mathbf{K}_{T T}
\end{array}\right]\left\{\begin{array}{l}
\mathbf{X}_{V} \\
\mathbf{X}_{T}
\end{array}\right\}=\left[\begin{array}{l}
\mathbf{F}_{V} \\
\mathbf{F}_{T}
\end{array}\right],
\end{aligned}
$$

in which the subscripts " $V$ " and " $T$ " denote the subsystems of the vehicle and the track, respectively; $\mathbf{M}, \mathbf{C}$, and $\mathbf{K}$ denote the mass, damping, and stiffness matrix, respectively; $\mathbf{F}$ denotes the loading vector.
In equation (9), the details of dynamic matrices in the equations of motion have been presented in [6].

Besides, the interactions between the vehicle and the tracks are mainly transmitted through the wheel-rail interfaces, where the wheel-rail contacts/creepages execute complex motions.

In this work, the wheel-rail nonlinear contacts at threedimensional (3D) space are depicted. A wheel-rail coupling model [1] is introduced, as shown in Figure 4. In this model, the relative motions between the full degrees of a wheelset and the vertical, lateral, and angular motions of the rail are accurately constructed from geometries and algebraic equations with nonlinearity.

The dynamic loads derived by this wheel-rail coupling model have been listed in [15].

2.3. Simple Comparisons with FASTSIM. Wheel-rail interaction forces have been known as the key technique of vehicle-track dynamics, which is also an aspect that is notoriously difficult to quantify theoretically. In this part, a comparison between this dynamic model and FASTSIM by Kalker [24] is presented with respect to wheel-rail forces, where track irregularities are used as system excitations.

Figure 5 shows the comparisons of wheel-rail longitudinal and lateral forces, respectively. It can be clearly observed from Figure 5 that the wheel-rail forces derived by this model agree well with the results of FASTSIM. Accordingly, the time-varying process of wheel-rail interactions including amplitude distribution and frequency components can be wholly demonstrated. Further validations about this vehicle-track interaction model have been presented in [6].

\section{A General Method to Estimate the Status of the Tracks}

The reality of track parameters can be expressed as

$$
\mathbf{X}_{k}:=\mathbf{x}_{k}^{[1]}, \mathbf{x}_{k}^{[2]}, \ldots, \mathbf{x}_{k}^{[N]},
$$

with $\mathbf{x}_{k}=\left(x_{k, 1}, x_{k, 2}, \ldots, x_{k, m}, \ldots\right)$, where the subscript " $k$ " denotes the time point, " $m$ " denotes the $m$ th track parameter, and there are $N$ spatial values for each track parameter vector $\mathbf{x}_{k, j}, j=1,2, \ldots, m$.

The state-space equations of the vehicle-track system can be further written by

$$
\mathbf{Z}_{k}(t \mid \mathbf{X})=H\left(\mathbf{X}_{k}, u_{k}, w_{k}\right), \quad t=\left[0, t_{t o l}\right] .
$$

In the above equation, $t$ denotes the time-varying process of the system responses; $H[\cdot]$ denotes the measurement function, which will be substituted by the vehicletrack model here. Thus, $\mathbf{Z}_{k}$ will denote the system responses. $u_{k}$ denotes other system factors; $w_{k}$ represents noise signals.

Obviously, it is a difficult work to measure the real status of tracks, especially with large scales. One is therefore expected to inversely and approximately estimate track 


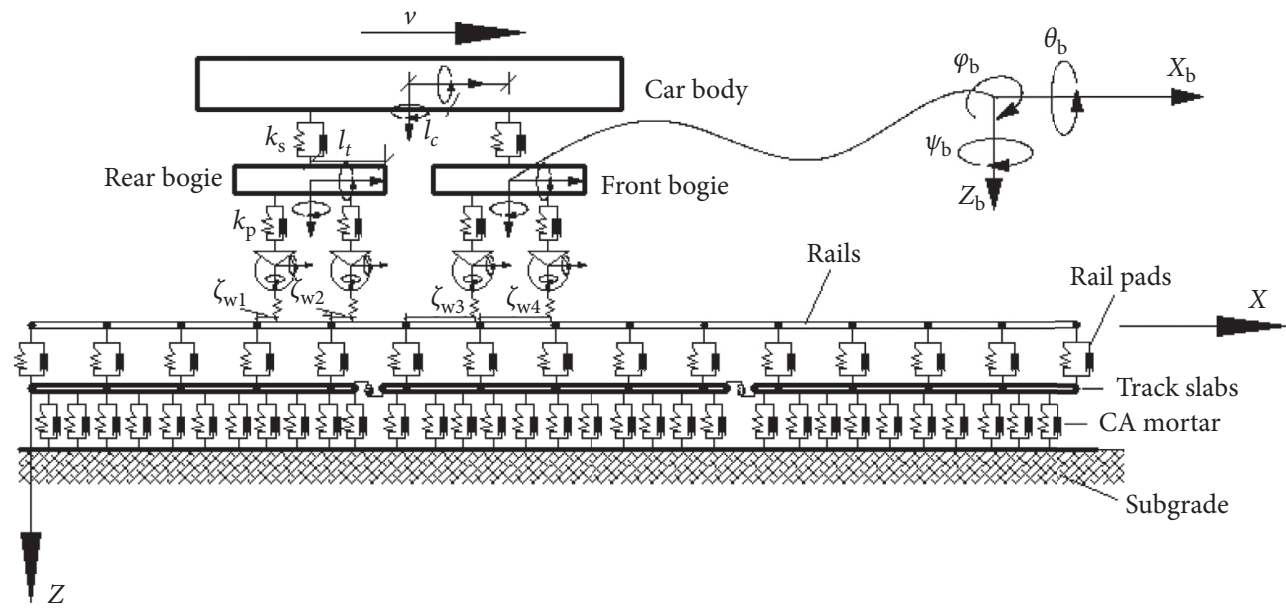

(a)

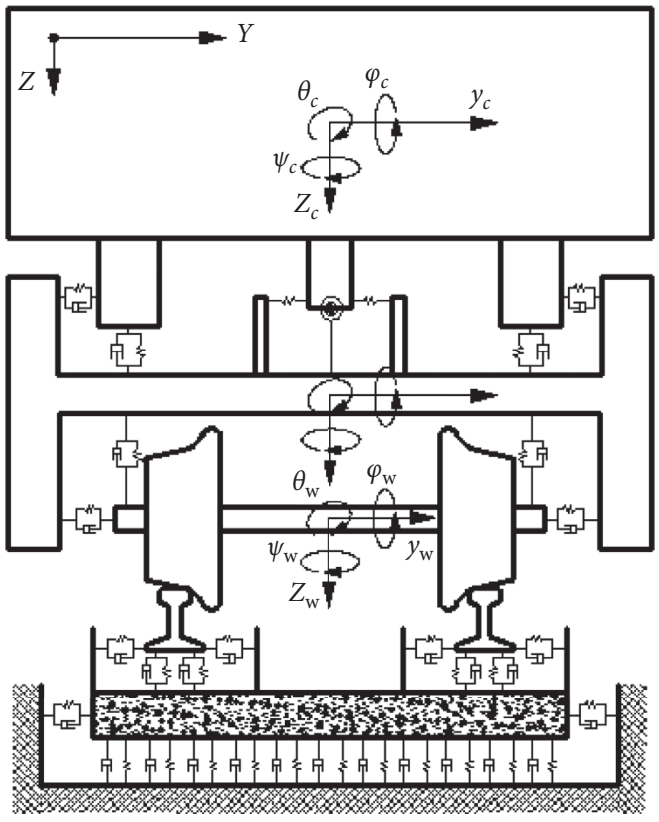

(b)

Figure 3: Vehicle-track 3D model. (a) Side view; (b) end view [22].

status (the distributions of track parameters) from the conventional dynamic responses and estimated status, namely, $\mathbf{Z}_{k}$ and $\mathbf{X}_{k-1}$.

The system responses are mainly induced by two factors: wheel/rail irregularities and inconstant mechanical-physical properties longitudinally and evolved. Therefore, the noise in equation (11) contains both the measurement error and the effects of irregularities.

In this present study, an efficient vehicle-track interaction model, denoted by $\wp$, had been fully constructed, where the physical-mechanical behaviors and interactions of the vehicle-track systems can be accurately depicted. The system responses derived by the theoretical model can therefore be calculated by

$$
\widetilde{\mathbf{Z}}_{k}=\wp\left(\mathbf{X}_{k}, \mathbf{\Omega}\right),
$$

where $\Omega$ denotes other system parameters including wheel/ rail irregularities.

Now a problem will be firstly encountered: we have no idea what the longitudinal distribution of the track parameter looks like. By applying a multi-correlated-variable Monte Carlo method [15], we can randomly generate a pseudoparameter matrix:

$$
\mathbf{M}_{k \mid \mathbf{X}}=\left[\begin{array}{cccc}
\mathbf{x}_{1,1}^{s} & \mathbf{x}_{1,2}^{s} & \ldots & \mathbf{x}_{1, N}^{s} \\
\mathbf{x}_{2,1}^{s} & \mathbf{x}_{2,2}^{s} & \ldots & \mathbf{x}_{2, N}^{s} \\
\vdots & \vdots & \ddots & \vdots \\
\mathbf{x}_{V, 1}^{s} & \mathbf{x}_{V, 2}^{s} & \ldots & \mathbf{x}_{V, N}^{s}
\end{array}\right]_{(V \times N) \times m}
$$

with $\ell\left(\mathbf{x}_{p, g}^{s}, \mathbf{x}_{p, h}^{s}\right)=R_{1}^{m}-\left(|g-h|\left(R_{1}^{m}-R_{0}^{m}\right) / N\right)$, in which the superscript "s" denotes the simulated value; $V$ denotes the total number of samples; $R_{0}^{m}$ and $R_{1}^{m}$ denote the 


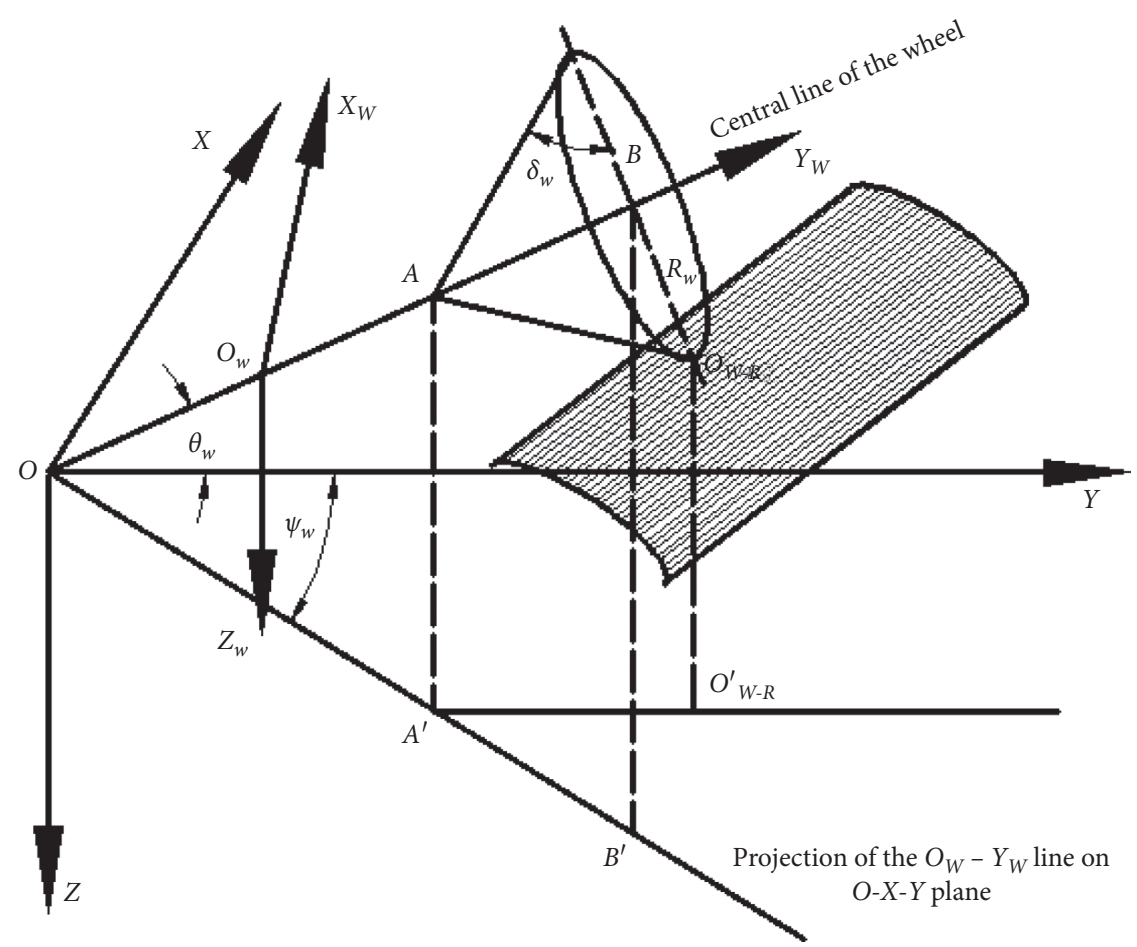

FIGURE 4: Wheel-rail solid contact model [6].

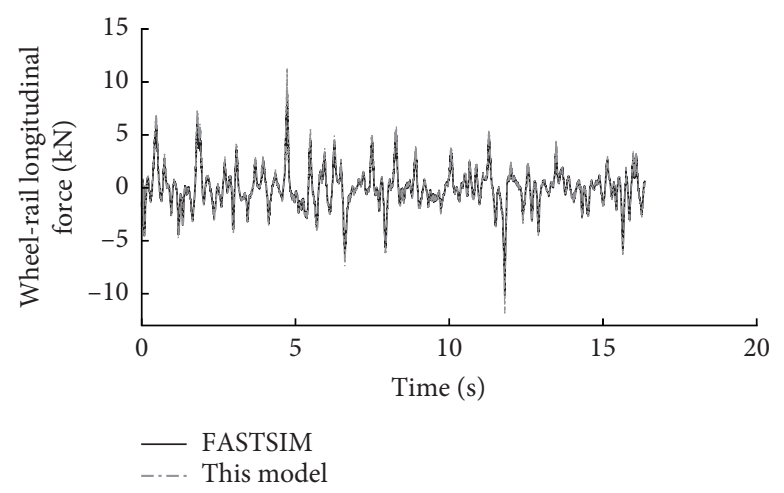

(a)

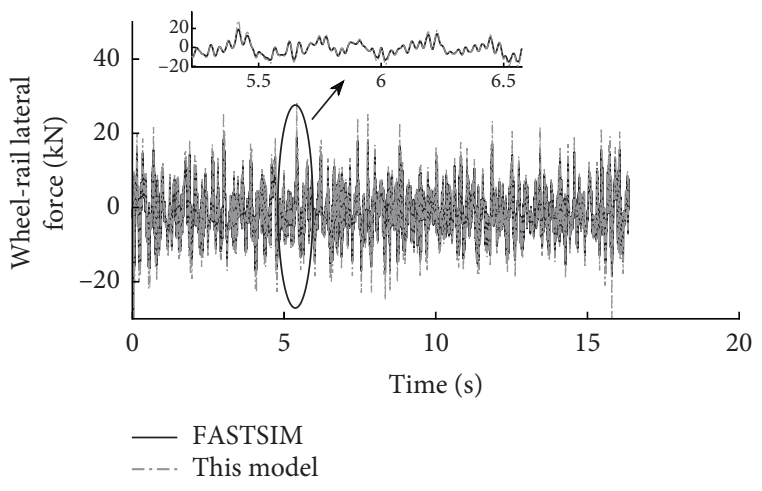

(b)

FIGURE 5: Comparison between FastSim and this dynamic model. (a) Wheel-rail longitudinal force; (b) wheel-rail lateral force.

minimum and maximum correlation coefficients for the $m$ th parameter, respectively; $\ell\left(\mathbf{x}_{p, q}^{s}, \mathbf{x}_{p, h}^{s}\right)$ denotes the correlation coefficient between the $g$ th and the $h$ th parameter columns.

Loading these $V$ parameter samples into the vehicletrack interaction model, one can obtain the system response matrix consequently; that is,

$$
\widetilde{\mathbf{Z}}_{k}\left(t \mid \mathbf{M}_{\mathbf{X}}\right)=\wp\left(\mathbf{M}_{k \mid \mathbf{X}}, \mathbf{\Omega}\right) .
$$

In reality, the real track parameters follow a specific probability distribution, namely, $\mathbf{x}_{k} \sim f_{\mathbf{x}, k}(x)$, and $f(x)$ is the probability function of the parameter $k$. Thus, the key work lies in building up the relationship between $f_{\mathbf{x}, k}(x)$, $\widetilde{Z}_{k}\left(t \mid \mathbf{M}_{\mathbf{X}}\right)$, and $\mathbf{Z}_{k}(t \mid \mathbf{X})$ and estimating $f_{\mathbf{x}, k}(x)$.
In stochastic analysis, we more likely intend to clarify the distribution of a variable in probability rather than attempting to estimate the deterministic values. Following the basic principle, the closer the correlation of system responses between simulation and measurement is, the more approachable to reality system parameters used in simulation are.

The steps below are therefore designed to achieve the estimation of $f_{\mathrm{x}, k}(x)$ :

(1) To increase the precision of estimation, the comparison between responses of calculation and measurement, that is, $\widetilde{Z}_{k}\left(t \mid \mathbf{M}_{\mathbf{X}}\right)$ and $\mathbf{Z}_{k}(t \mid \mathbf{X})$, are conducted within multiple time segments, $\Delta t_{a}=t_{\mathrm{tol}} \cdot[((a-1) / A),(a / A)], a=1,2, \ldots, A$. 
(2) The uncertainty of the responses will be estimated by the correlation between the calculation and the measurement at time portion $\Delta t_{a}$.

$$
W_{\tau}^{k}(a)=\frac{\operatorname{cov}\left(\widetilde{\mathbf{Z}}_{k}\left(\Delta t_{a} \mid \mathbf{M}_{\mathbf{X}}\right), \mathbf{Z}_{k}\left(\Delta t_{a} \mid \mathbf{X}\right)\right)}{\sigma_{\widetilde{\mathbf{Z}}_{k, \Delta t_{a}}} \sigma_{\mathbf{Z}_{k, \Delta t_{a}}}},
$$

where the subscript " $\tau$ " denotes the type of the responses, calculation, or measurement: $\tau=\widetilde{Z}_{k}$ or $\mathbf{Z}_{k}$, respectively; $z_{a}$ denotes the system responses, $z_{a} \in \widetilde{Z}_{k}\left(t \mid \mathbf{M}_{\mathbf{X}}\right)$ or $\mathbf{Z}_{k}(t \mid \mathbf{X})$; $\operatorname{cov}(\cdot)$ denotes the estimator of covariance and $\sigma$ denotes the standard deviation. Obviously, $W_{\widetilde{\mathbf{Z}}_{k}}^{k}(a)$ is a matrix with order of $V \times A$, while $W_{\mathbf{Z}_{k}}^{k}(a)$ is a matrix with order of $1 \times A$.

(3) The weight coefficients evaluating the contribution of the parameters on system responses can be obtained by

$$
\gamma_{l, a}^{k}(x)=\frac{1}{\sum_{l=1}^{V} w_{l}} \sum_{l=1}^{V} w_{l} \delta[x-l]
$$

with $\quad w_{l, a}=p\left(z_{l, a} \mid \mathbf{x}_{l, a}\right) \propto\left(1 / \sqrt{2 \pi Q^{(1 / 2)}}\right)$ $e^{-\left(\left(W_{Z_{k}}^{k}(l, a)-W_{z_{k}}^{k}(a)\right)^{2} / 2 Q\right)}$

e $z_{k} \quad, l=1,2, \ldots, V$, where $Q$ is the variance of the measurement noise, $Q=0.05 \sim 0.4$, and $p(z \mid \mathbf{x})$ is the posterior distribution of the response under certain track conditions.

(4) Based on equation (16), the estimated distribution of the parameters will be assessed by

$$
\tilde{f}_{\mathbf{x}, k}(x)=\sum_{a} \sum_{l} \gamma_{l, a}^{k}(x) f_{l, a \mid \mathbf{M}_{k \mid \mathbf{X}}}(x),
$$

where $f_{l, a \mid \mathbf{M}_{k \mid \mathbf{X}}}(x)$ indicates the probability distribution of the parameters in the parameter matrix.

Finally, the estimation of $f_{\mathbf{x}, k}(x)$ can be approximated by assuming $f_{\mathbf{x}, k}(x) \cong \widetilde{f}_{\mathbf{x}, k}(x)$.

Since $f_{\mathbf{x}, k}(x)$ has been estimated approximately, an acceptance-rejection algorithm, as illustrated in Algorithm 1 , can be introduced to randomly simulate the spatial series of track parameters $\mathbf{X}_{k}$.

\section{Numerical Examples}

In the numerical examples, it is assumed that the vehicle runs with a constant velocity of $300 \mathrm{~km}$ per hour on the tracks. The main parameters of the vehicle and the tracks are listed in Tables 1 and 2, respectively [1].

4.1. Influence of the Longitudinal Inhomogeneity of Track Stiffness on System Responses. To clearly reveal the dynamic influence of the longitudinal inhomogeneity of track stiffness on system responses, track profile irregularities will not be taken into account in the vehicle-track interactions in this subsection. It is assumed that the values of a track parameter satisfying specific probability characteristics are randomly distributed along the railway line instead of a constant.

Three types of probability distributions are considered: uniform distribution $U_{\mathbf{X}}$, normal distribution $N_{\mathbf{X}}$, and $\log$ normal distribution $\log -N_{\mathbf{X}}$. To guarantee the comparability for different conditions of property inhomogeneity, the random parameter values should be within almost the same ranges. The equations below are therefore satisfied:

$$
\left\{\begin{array}{l}
F_{U_{\mathbf{x}}}^{-1}(0.01)=F_{N_{\mathbf{x}}}^{-1}(0.01)=F_{\log -N_{\mathbf{x}}}^{-1}(0.01), \\
F_{U_{\mathbf{x}}}^{-1}(0.99)=F_{N_{\mathbf{x}}}^{-1}(0.99)=F_{\log -N_{\mathbf{x}}}^{-1}(0.99)
\end{array}\right.
$$

where $F[\cdot]$ denotes the cumulative probability function and the superscript "-1" denotes the inverse function.

Take the response distribution of car body accelerations, wheel-rail forces, displacements, and accelerations of the rail and the track slab as examples. For normal distributions, the coefficient of variation (CV) is denoted by

$$
\mathrm{CV}=\frac{\sigma}{\mu} \text {, }
$$

where $\sigma$ is the standard deviation and $\mu$ is the mean. $\mathrm{CV}=$ 0.2 in this example.

Figure 6 illustrates the statistical mean PDF of wheel-rail vertical force, rail vertical displacement, and track-slab vertical displacement with 200 samples simulated by different forms of distributions of rail pad vertical stiffness. From Figure 6, one can observe that the longitudinal variability of rail pad vertical stiffness shows a significant influence on the probabilistic distribution of system vertical vibrations. Besides the variation of divergence and response amplitude of a specific index accompanied by the changes of the parametric distribution, the longitudinal uniform distribution exerts more effects on the variance of system responses compared to the other two, normal distribution and log-normal distribution.

For quantitatively characterizing the uncertainty of a random signal, the concept of entropy can be introduced by

$$
H(z)=E[-\ln (p(z))]
$$

where $H$ denotes the entropic value, $E[\cdot]$ denotes the mathematical expectation, $\ln (\cdot)$ denotes the logarithm with bottom $e$, and $p(z)$ denotes the probability function.

As an example, Figure 7 shows the entropic distribution of the wheel-rail vertical force, from which one can observe the following: first, the uncertainty of the wheel-rail vertical force excited by the uniformly distributed rail pad vertical stiffness is significantly higher than those excited by normal and log-normal distributions; second, it can be observed from Figure $7(\mathrm{~b})$ that the statistical uncertainty of system responses fluctuates slightly, which means that once the parametric distribution is confirmed, we can approximately estimate its influence on the distribution of system responses.

Figure 8 further plots the PDF of wheel-rail lateral force and rail lateral displacement affected by the deviations of rail pad lateral stiffness. In this example, for showing the influence of the magnitude of rail pad lateral stiffness on 


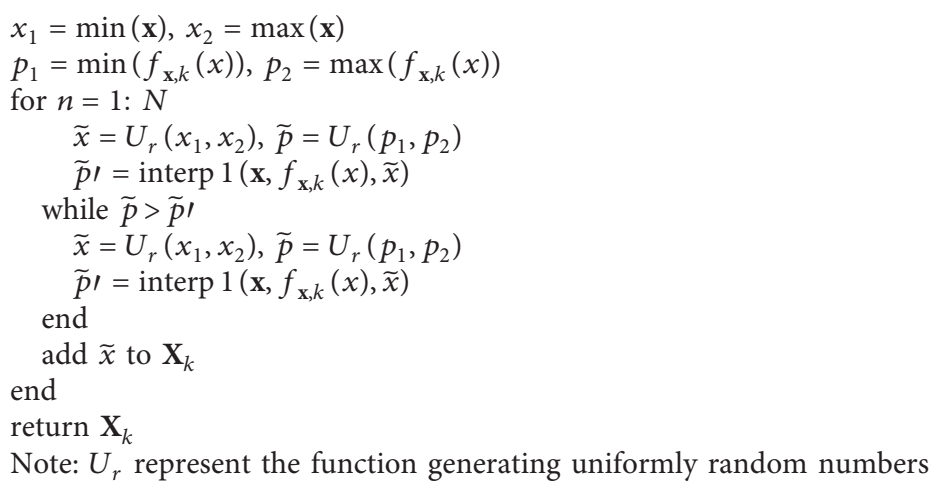

Algorithm 1: Acceptance-rejection algorithm for simulating spatial parameters with longitudinal inhomogeneity.

TABLE 1: Main parameters of the railway vehicles used in the simulation.

\begin{tabular}{|c|c|c|}
\hline Notation & Parameter & Value \\
\hline$M_{c}$ & Car body mass $(\mathrm{kg})$ & 46280 \\
\hline$M_{t}$ & Bogie mass $(\mathrm{kg})$ & 3300 \\
\hline$M_{w}$ & Wheelset mass $(\mathrm{kg})$ & 1780 \\
\hline$I_{c x}$ & Mass moment of the inertia of the car body about the $X$-axis $\left(\mathrm{kg} \mathrm{m}^{2}\right)$ & 149970 \\
\hline$I_{c y}$ & Mass moment of the inertia of the car body about the $Y$-axis $\left(\mathrm{kg} \mathrm{m}^{2}\right)$ & 2267765 \\
\hline$I_{c z}$ & Mass moment of the inertia of the car body about the $Z$-axis $\left(\mathrm{kg} \mathrm{m}^{2}\right)$ & 2139900 \\
\hline$I_{t x}$ & Mass moment of the inertia of the bogie about the $X$-axis $\left(\mathrm{kg} \mathrm{m}^{2}\right)$ & 2673 \\
\hline$I_{t y}$ & Mass moment of the inertia of the bogie about the $Y$-axis $\left(\mathrm{kg} \mathrm{m}^{2}\right)$ & 1807 \\
\hline$I_{t z}$ & Mass moment of the inertia of the bogie about the $Z$-axis $\left(\mathrm{kg} \mathrm{m}^{2}\right)$ & 3300 \\
\hline$I_{w x}$ & Mass moment of the inertia of the wheelset about the $X$-axis $\left(\mathrm{kg} \mathrm{m}^{2}\right)$ & 949 \\
\hline$I_{w y}$ & Mass moment of the inertia of the wheelset about the $Y$-axis $\left(\mathrm{kg} \mathrm{m}^{2}\right)$ & 118 \\
\hline$I_{w z}$ & Mass moment of the inertia of the wheelset about the $Z$-axis $\left(\mathrm{kg} \mathrm{m}^{2}\right)$ & 967 \\
\hline$K_{p x}$ & Stiffness coefficient of the primary suspension along the $X$-axis $(\mathrm{MN} / \mathrm{m})$ & 14.68 \\
\hline$K_{p y}$ & Stiffness coefficient of the primary suspension along the $Y$-axis $(\mathrm{MN} / \mathrm{m})$ & 6.47 \\
\hline$K_{p z}$ & Stiffness coefficient of the primary suspension along the $Z$-axis $(\mathrm{MN} / \mathrm{m})$ & 1.176. \\
\hline$K_{s x}$ & Stiffness coefficient of the secondary suspension along the $X$-axis $(\mathrm{MN} / \mathrm{m})$ & 0.167 \\
\hline$K_{s y}^{s i n}$ & Stiffness coefficient of the secondary suspension along the $Y$-axis $(\mathrm{MN} / \mathrm{m})$ & 0.167 \\
\hline$K_{s z}$ & Stiffness coefficient of the secondary suspension along the $Z$-axis $(\mathrm{MN} / \mathrm{m})$ & 0.323 \\
\hline$C_{p z}$ & Damping coefficient of the primary suspension along the $Z$-axis $(\mathrm{kN} \mathrm{s} / \mathrm{m})$ & 9.8 \\
\hline$C_{s y}$ & Damping coefficient of the secondary suspension along the $Y$-axis $(\mathrm{kN} \mathrm{s} / \mathrm{m})$ & 39.2 \\
\hline$C_{s z}$ & Damping coefficient of the secondary suspension along the $Z$-axis $(\mathrm{kN} \mathrm{s} / \mathrm{m})$ & 9.8 \\
\hline$L_{c}$ & Semilongitudinal distance between bogies $(\mathrm{m})$ & 8.75 \\
\hline$L_{t}$ & Semilongitudinal distance between wheelsets in a bogie $(\mathrm{m})$ & 1.25 \\
\hline$R_{0}$ & Wheel radius $(\mathrm{m})$ & 0.43 \\
\hline
\end{tabular}

system lateral vibrations, the stiffness coefficients following log-normal distribution are given higher values, as shown in Figure 9.

It can be observed from Figure 8 that the longitudinal inhomogeneity of rail pad lateral stiffness also shows distinctive effects on the distribution characteristics of the wheel-rail lateral force and the rail pad lateral displacement. Obviously, the larger the lateral stiffness of the rail pad, the smaller the wheel-rail lateral force, and the larger the rail lateral displacement. It is similar to the results derived above where the uniform distribution shows higher influence on the uncertainty of system response compared to the other two distributions.

In Figure 10, the PDF of rail vertical displacement is exposed, from which one can observe that the longitudinal distribution of the rail pad lateral stiffness has little influence on the system's vertical vibrations.

The results for the dynamic response induced by the longitudinal inhomogeneity of vertical stiffness of the cement asphalt mortar (CAM) (subgrade support layer) are shown in Figure 11. The vertical stiffness of the CAM shows great influence on the responses of track components, for example, the rails and the track slabs. However, its influence on the wheel-rail interaction forces is relatively small.

4.2. Estimation of the Distribution of Track Parameters. From the numerical analysis above, one can recognize that the rail pad stiffness, especially the vertical stiffness, shows significant influence on wheel-rail forces and rail 
TABLE 2: Main parameters of the railway tracks used in the simulation.

\begin{tabular}{lcc}
\hline Notation & Parameter \\
\hline$E$ & Elastic modulus of the rail $\left(\mathrm{N} / \mathrm{m}^{2}\right)$ & Value \\
$\bar{m}_{r}$ & Mass of the rail per unit length $(\mathrm{kg} / \mathrm{m})$ & $2.059 \times 10^{11}$ \\
$I_{0}$ & Torsional inertia of the rail $\left(\mathrm{m}^{4}\right)$ & 6.64 \\
$I_{y}$ & Rail second moment of the area about the $Y$-axis $\left(\mathrm{m}^{4}\right)$ & $3.741 \times 10^{-5}$ \\
$I_{z}$ & Rail second moment of the area about the $Z$-axis $\left(\mathrm{m}^{4}\right)$ & $3.217 \times 10^{-5}$ \\
$k_{z}^{\mathrm{rp}}$ & Fastener stiffness in the vertical direction $(\mathrm{N} / \mathrm{m})$ & $5.24 \times 10^{-6}$ \\
$k_{y}^{\mathrm{rp}}$ & Fastener stiffness in the lateral direction $(\mathrm{N} / \mathrm{m})$ & $4.0 \times 10^{7}$ \\
$c_{z}^{r \text { p }}$ & Fastener damping in the vertical direction $(\mathrm{N} \mathrm{s} / \mathrm{m})$ & $1.5 \times 10^{7}$ \\
$c_{y}^{\mathrm{rp}}$ & Fastener damping in the lateral direction $(\mathrm{N} \mathrm{s} / \mathrm{m})$ & $5.0 \times 10^{4}$ \\
$k_{y}^{\text {cam }}$ & Equivalent lateral stiffness coefficient of the CAM $(\mathrm{N} / \mathrm{m})$ & $5.0 \times 10^{4}$ \\
$k_{z}^{\text {cam }}$ & Equivalent vertical stiffness coefficient of the CAM $(\mathrm{N} / \mathrm{m})$ & $6.5 \times 10^{7}$ \\
$c_{y}^{\text {cam }}$ & Equivalent lateral damping coefficient of the CAM $(\mathrm{N} \mathrm{s} / \mathrm{m})$ & $2.4 \times 10^{8}$ \\
$c_{z}^{\text {cam }}$ & Equivalent vertical damping coefficient of the CAM $(\mathrm{N} \mathrm{s} / \mathrm{m})$ & $3.1 \times 10^{4}$ \\
\hline
\end{tabular}

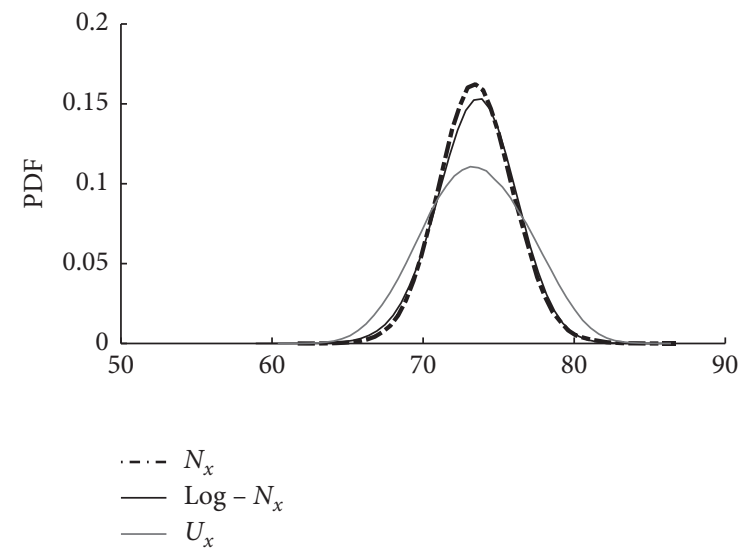

(a)

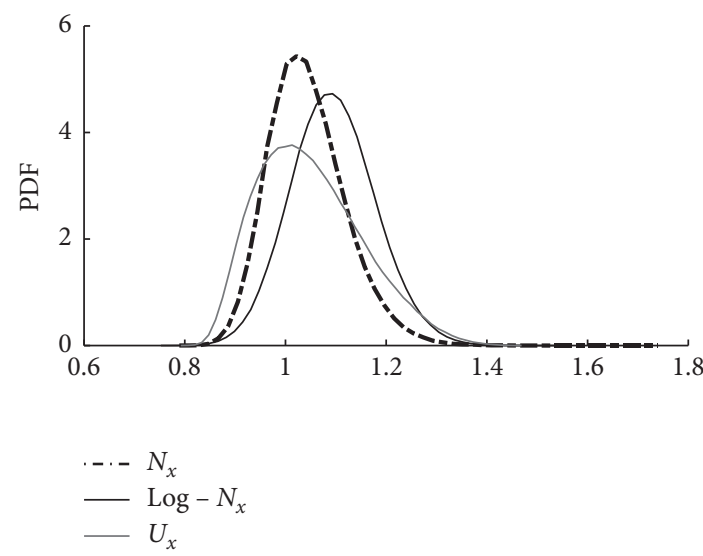

(b)

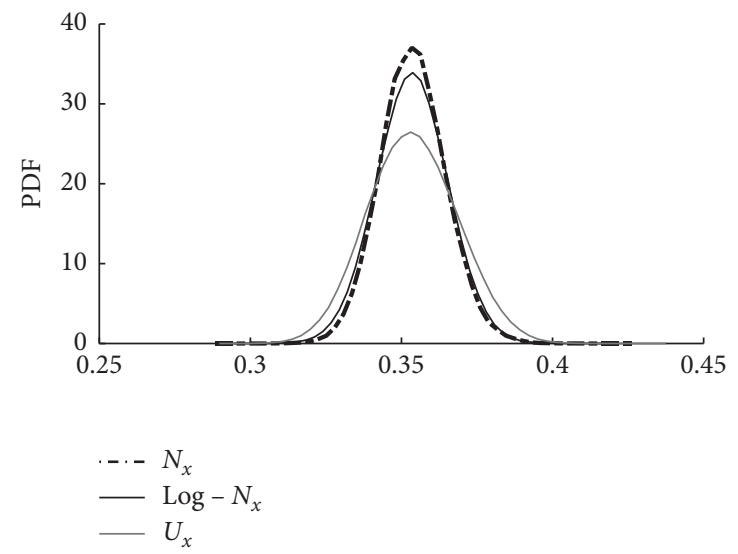

(c)

FIgURE 6: The PDF of dynamic indices specific to different longitudinal distribution of rail pad vertical stiffness. (a) Wheel-rail vertical force; (b) rail vertical displacement; (c) track-slab vertical displacement.

vertical displacement. Hence, we therefore put an emphasis on estimation of the distribution of rail pad vertical stiffness.

The track irregularities used in vehicle-slab track interactions from a high-speed line are shown in Figure 12. Besides, the longitudinally nonuniform rail pad vertical stiffness is assumed to follow normal distribution with $\mathrm{CV}$ of $0.05,0.10$, and 0.20 .
In the estimations, the following computational conditions are considered:

(1) Only track irregularities are loaded as the system excitations, where the system responses are denoted by $\mathbf{Z}(t \mid \Omega)$

(2) Track irregularities and randomly distributed rail pad vertical stiffness (which is assumed as the real 


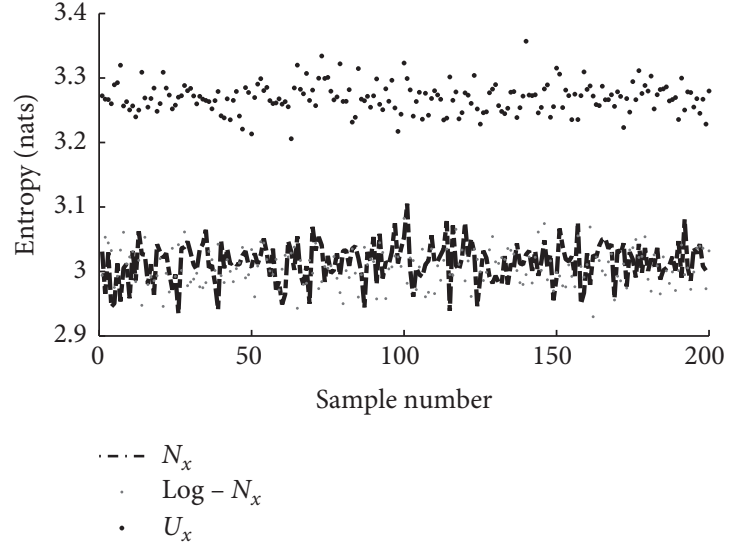

(a)

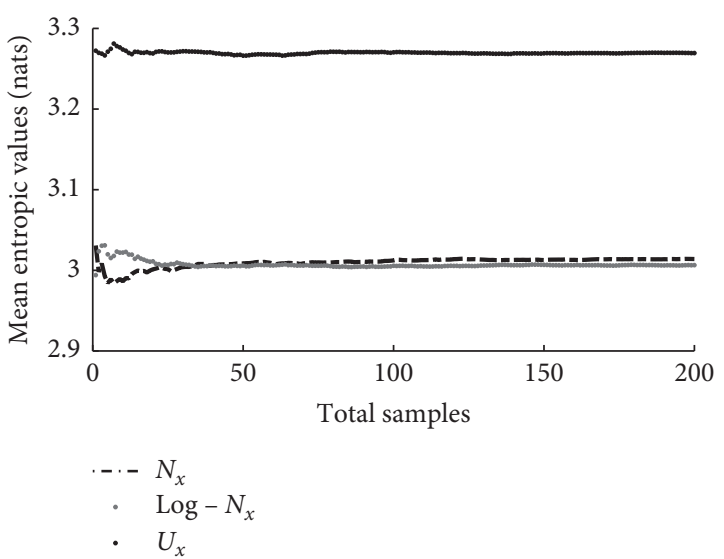

(b)

Figure 7: The entropy of PDF of wheel-rail vertical force due to variation of rail pad vertical stiffness. (a) Entropic value for specific samples; (b) the statistical mean value of entropy under different total samples.

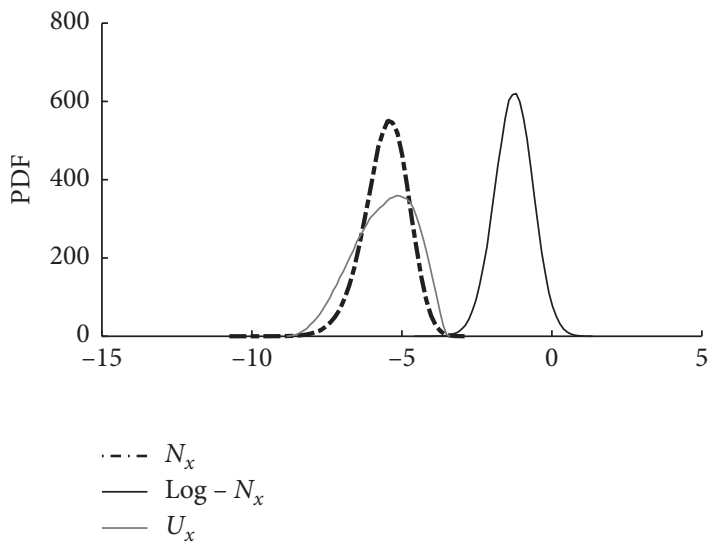

(a)

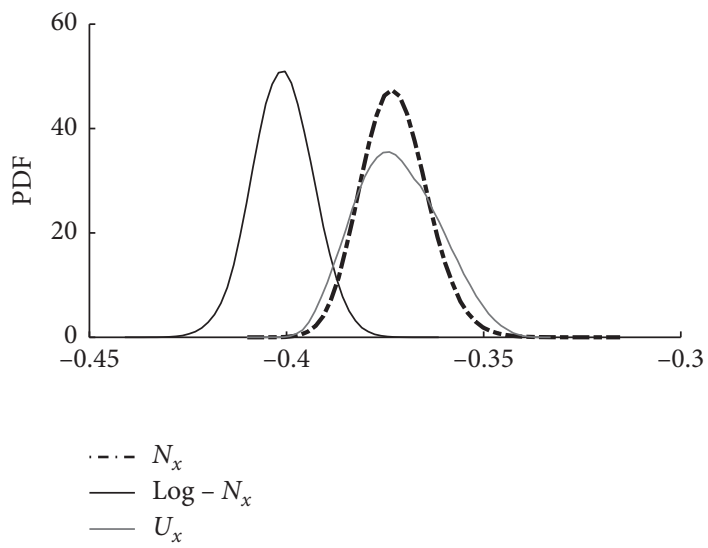

(b)

FIGURE 8: PDF of dynamic indices due to longitudinal inhomogeneity of rail pad lateral stiffness. (a) Rail lateral displacement; (b) wheel-rail lateral displacement.

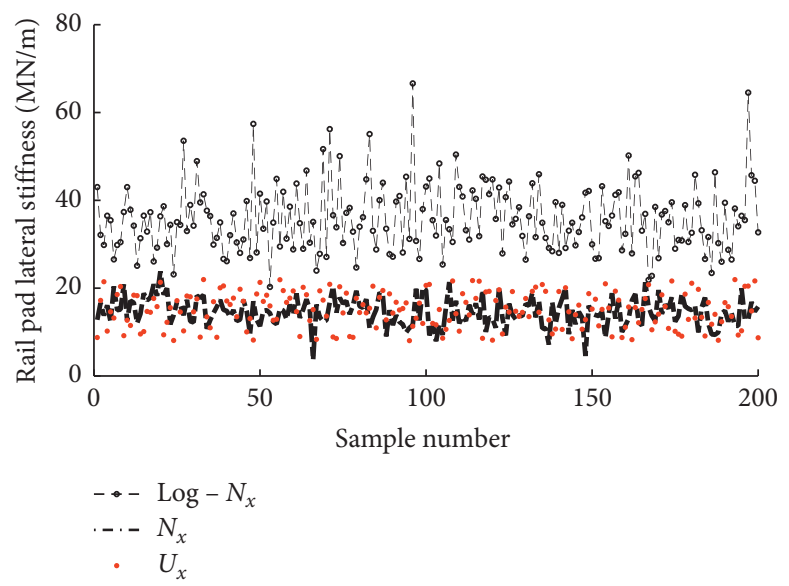

Figure 9: The lateral stiffness of 200 samples for a specific rail pad. 


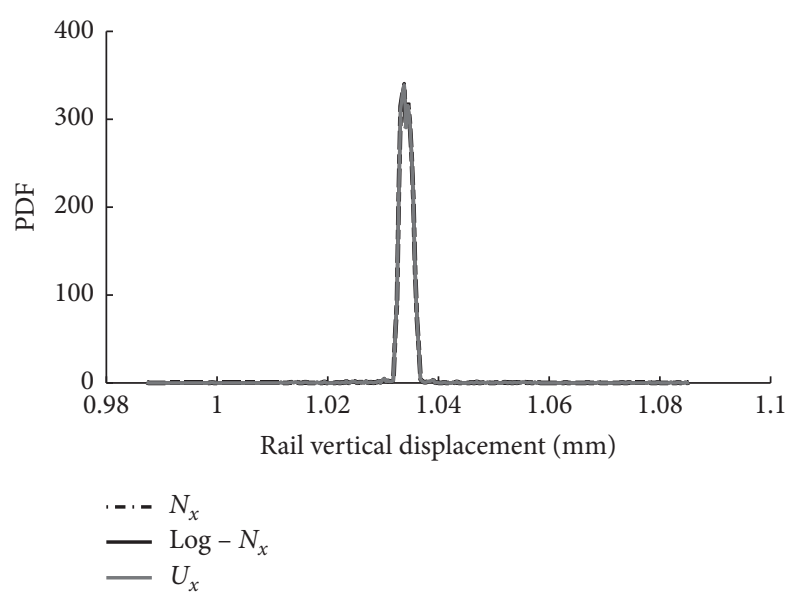

Figure 10: The influence of longitudinal distribution of rail pad lateral stiffness on the distribution of the rail vertical displacement.

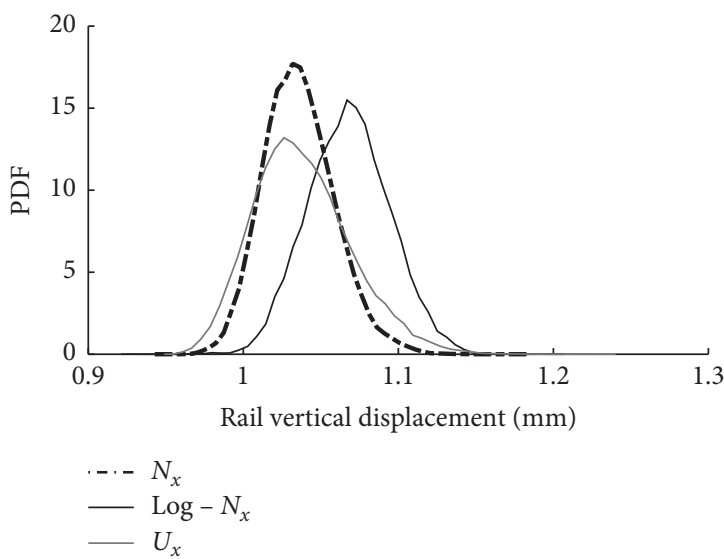

(a)

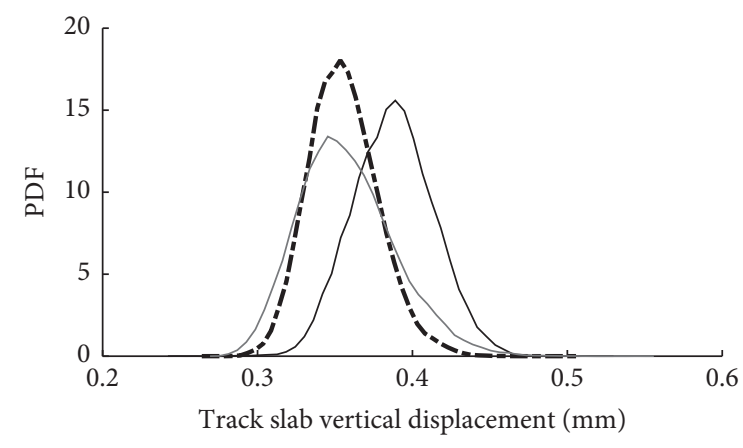

... $N_{x}$

$-\log -N_{x}$

$-U_{x}$

(b)

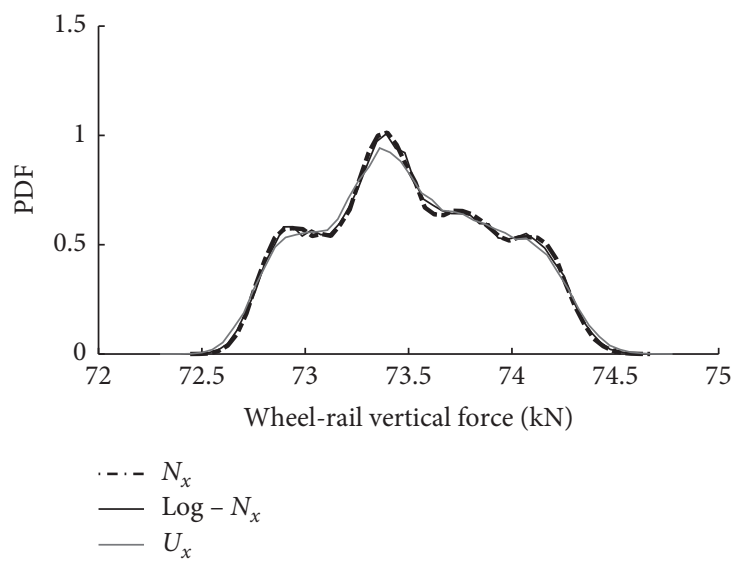

(c)

Figure 11: The PDF of dynamic responses due to the nonuniformity of vertical stiffness of CAM. (a) Rail vertical displacement (mm); (b) track-slab vertical displacement $(\mathrm{mm})$; (c) wheel-rail vertical force $(\mathrm{kN})$.

distribution of "measurement" in the theoretical analysis) are loaded as the system excitations, where the responses are denoted by $\mathbf{Z}(t \mid \mathbf{X})$
(3) The responses excited by a group of correlated variables of rail pad stiffness and the specific track irregularities are denoted by $\mathbf{Z}\left(t \mid \mathbf{M}_{\mathbf{X}}\right)$ 


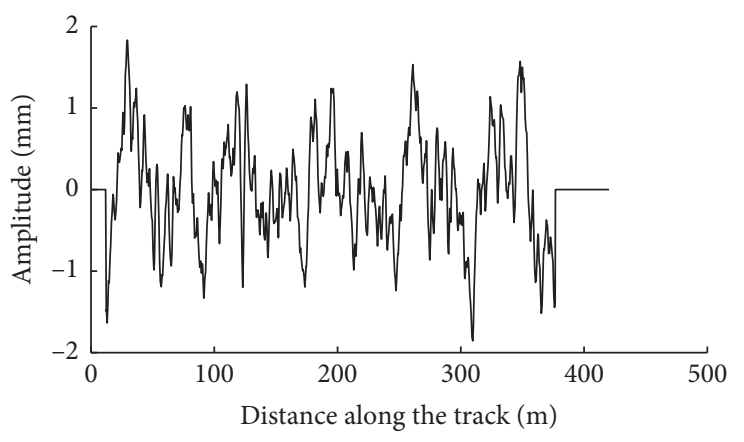

(a)

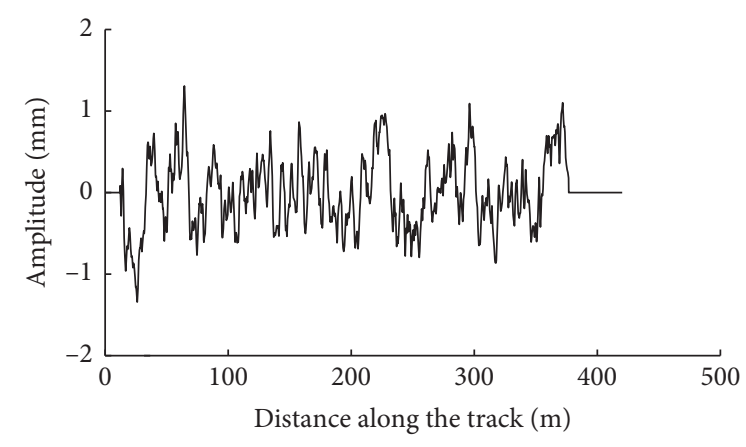

(b)

FIgURe 12: Track irregularities used in the dynamic model. (a) Vertical irregularity; (b) lateral irregularity.

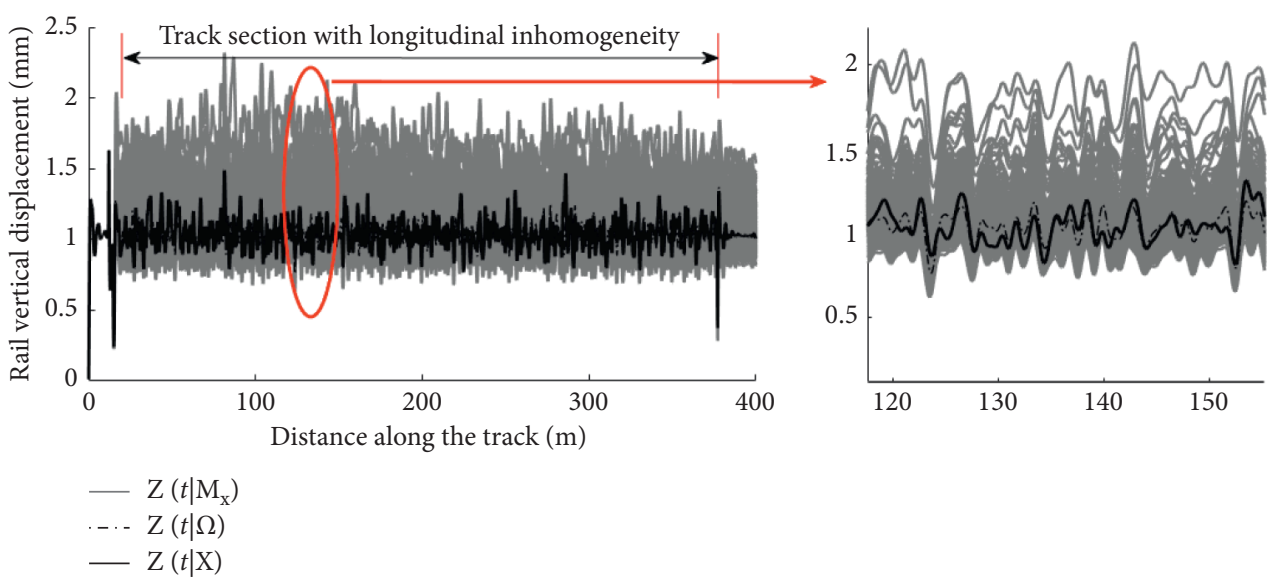

Figure 13: The responses of rail vertical displacement due to different excitations with CV of rail pad vertical stiffness $=0.20$.

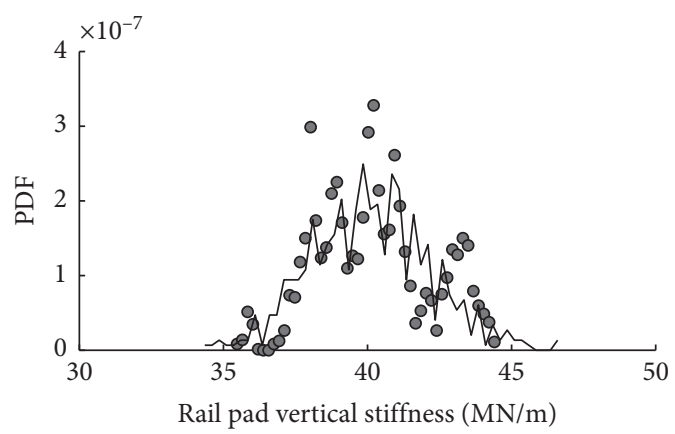

- Estimation

Assumption

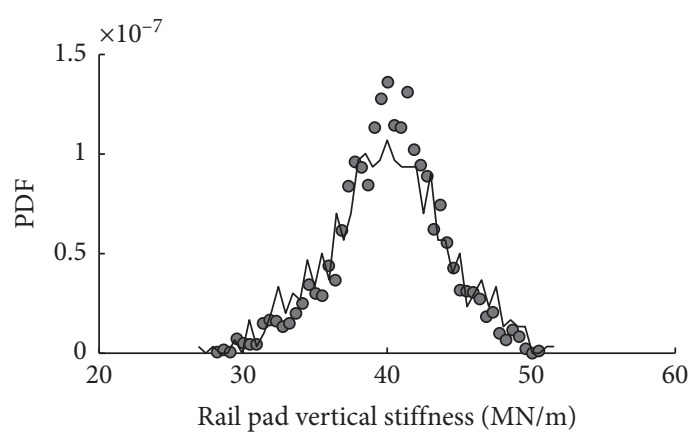

- Estimation Assumption

(a)

FIgURe 14: Continued. 


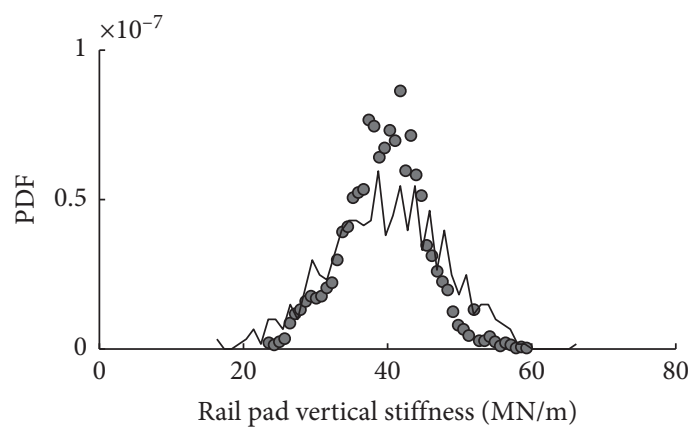

- Estimation

- Assumption

(c)

Figure 14: Comparisons between estimation and measurement estimated by the rail vertical displacement. (a) $\mathrm{CV}=0.05$; (b) $\mathrm{CV}=0.10$; (c) $\mathrm{CV}=0.20$

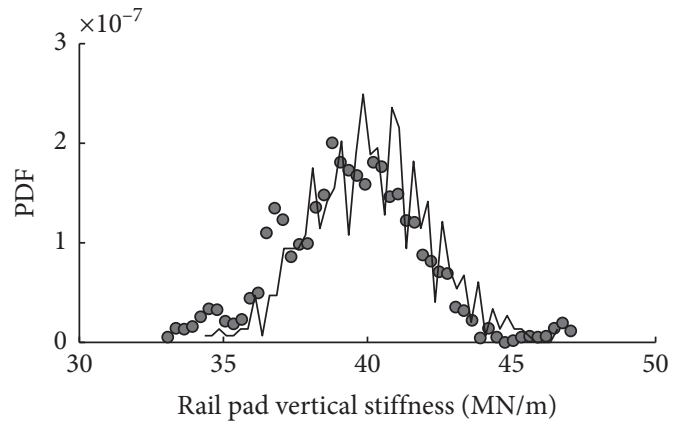

- Estimation - Assumption

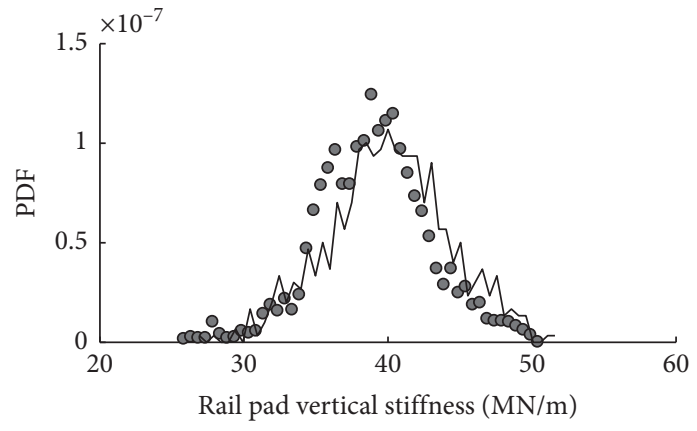

- Estimation

- Assumption

(a)

(b)

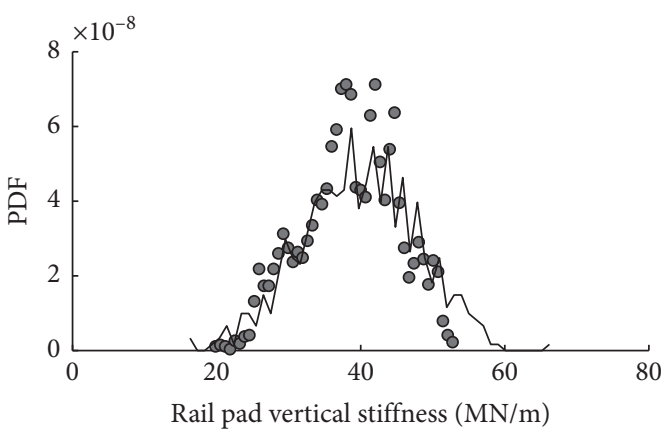

- Estimation

Assumption

(c)

Figure 15: Comparisons between estimation and measurement estimated by the wheel-rail vertical forces. (a) $C V=0.05$; (b) $C V=0.10$; (c) $\mathrm{CV}=0.20$

Figure 13 plots the time-domain responses of rail vertical displacement with $\mathrm{CV}=0.2$, from which one can observe that the rail vertical displacement changes significantly against time once with the longitudinal inhomogeneity of vertical stiffness of rail pads, that is, $\mathbf{Z}(t \mid \Omega)$ versus $\mathbf{Z}(t \mid \mathbf{X})$. The response assemblage, that is, $\mathbf{Z}\left(t \mid \mathbf{M}_{\mathbf{X}}\right)$, is the result of a set of excitations (parametric and irregularity), which has properly covered the responses $\mathbf{Z}(t \mid \Omega)$ and $\mathbf{Z}(t \mid \mathbf{X})$.

According to the method presented in Section 3, we can estimate the probability density function (PDF) of the vertical stiffness of the rail pad, as shown in Figure 14. As seen from Figure 14, the estimated distribution of the rail 
pad vertical stiffness coincides well with that of assumption in various variations: $C V=0.05,0.10$, and 0.20 . Through probability entropy calculations, the entropy difference between estimation and assumption is, respectively, 0.002, 0.01 , and 0.008 for conditions $\mathrm{CV}=0.05, \mathrm{CV}=0.10$, and $\mathrm{CV}=0.20$. From the analysis in Section 4.1, one has recognized that once the longitudinal distribution of a track parameter is determined, the system responses it caused can be approximately estimated with a slight deviation.

Undoubtedly, one can also estimate the distribution of the rail pad vertical stiffness through the wheel-rail vertical forces. The corresponding results are shown in Figure 15, from which it can be clearly observed that estimation of the stiffness distribution also approaches those of assumption.

From the numerical examples, it can be seen that the present method is highly effective. However, limitations have also been notified in its implementation to engineering practices; that is, the $\mathrm{CV}$ of track parameters is a prerequisite in the simulation. An abundant measurement data is therefore required to build the mapping relationship between system responses and track parametric distributions.

\section{Conclusions}

In this paper, the dynamic influences of the longitudinally distributed track parameters on system responses are systematically studied using stochastic and statistical approach, and a new approach for estimating the parametric distribution of the tracks was developed theoretically.

From the numerical studies, several conclusions can be drawn accordingly:

(1) The longitudinal inhomogeneity of track stiffness shows significant influence on vehicle-track interactions. Besides, the distribution characteristics of the system responses are varied against the difference of distribution of track stiffness.

(2) The mean entropy value of wheel-rail vertical force is increased by about $8 \%$ when it is assumed that the rail vertical stiffness follows uniform distribution instead of normal and log-normal distribution. Besides, it is observed that the response dispersion degree tends to converge when the random samples are larger than 50 .

(3) The nonuniformity of track vertical stiffness shows larger influence on wheel-rail forces and track structural deformations in comparison with the lateral stiffness.

(4) It could be a practical way to assess the effects of random distribution of track parameters on the uncertainty of system responses.

(5) The state estimation method newly developed in this work is fairly effective in estimating the track parametric distributions.

In the future, three aspects are urgently needed to study: the first is a more physically approachable model to simulate the vehicle-track dynamic performance with respect to spatial variability and nonlinearity of track properties, the second is the development of a highly accurate and efficient measurement method to detect the vehicle-track system responses, and the third is an exploration of practical methods to estimate the track parametric distribution through the vehicular responses in the condition of low signal-to-noise ratio.

\section{Data Availability}

Data will be available upon request to the corresponding author.

\section{Conflicts of Interest}

The authors declare that they have no conflicts of interest.

\section{Acknowledgments}

This work was supported by the National Natural Science Foundation of China (no. 52008404 and 52008382), Earthquake Research Fund of CEA (2019B01 and 2019C09), Heilongjiang Provincial Natural Science Foundation of China (no. LH2019E094), and Program for Innovative Research Team in China Earthquake Administration.

\section{References}

[1] W. Zhai, Vehicle-Track Coupled Dynamics, Science Press, Beijing, China, 4th edition, 2015.

[2] H. Fang, "Influence of pier settlement on structural stress of longitudinal connected track in high-speed railways," Advances in Civil Engineering, vol. 2020, Article ID 8102637, 2020.

[3] S. Zhu, J. Wang, C. Cai et al., "Development of a vibration attenuation track at low frequencies for urban rail transit," Computer-Aided Civil and Infrastructure Engineering, vol. 32, no. 9, pp. 713-726, 2017.

[4] G. Götz and O. Polach, "Verification and validation of simulations in a rail vehicle certification context," International Journal of Rail Transportation, vol. 6, no. 2, pp. 83-100, 2018.

[5] J. N. Varandas, A. Paixão, and E. Fortunato, "A study on the dynamic train-track interaction over cut-fill transitions on buried culverts," Computers \& Structures, vol. 189, pp. 49-61, 2017.

[6] L. Xu and W. Zhai, "A novel model for determining the amplitude-wavelength limits of track irregularities accompanied by a reliability assessment in railway vehicle-track dynamics," Mechanical Systems and Signal Processing, vol. 86, pp. 260-277, 2017.

[7] R. Zarfam and A. R. Khaloo, "Vibration control of beams on elastic foundation under a moving vehicle and random lateral excitations," Journal of Sound and Vibration, vol. 331, no. 6, pp. 1217-1232, 2012.

[8] L. Xu and X. Liu, "Matrix coupled model for the vehicle-track interaction analysis featured to the railway crossing," $M e$ chanical Systems and Signal Processing, vol. 152, no. 1, p. 107485, 2021.

[9] T. X. Wu and D. J. Thompson, "On the parametric excitation of the wheel/track system," Journal of Sound and Vibration, vol. 278, no. 4-5, pp. 725-747, 2004.

[10] B. Nélain and N. Vincent, "The effect of parametric excitation on ground borne vibration in railway applications," in 
Proceedings of 9th International Conference on Structural Dynamics (EURODYN 2014), pp. 847-853, Porto, Portugal, June 2014.

[11] R. Verachtert, H. E. M. Hunt, M. F. M. Hussein, and G. Degrande, "Changes of perceived unevenness caused by intrack vibration countermeasures in slab track," European Journal of Mechanics-A/Solids, vol. 65, pp. 40-58, 2017.

[12] J. Mao, Z. Yu, Y. Xiao, C. Jin, and Y. Bai, "Random dynamic analysis of a train-bridge coupled system involving random system parameters based on probability density evolution method," Probabilistic Engineering Mechanics, vol. 46, pp. 48-61, 2016.

[13] F. Lu, J. H. Lin, D. Kennedy, and F. W. Williams, "An algorithm to study non-stationary random vibrations of vehicle-bridge systems," Computers \& Structures, vol. 87, no. 3-4, pp. 177-185, 2009.

[14] A. Roghani and M. T. Hendry, "Quantifying the impact of subgrade stiffness on track quality and the development of geometry defects," Journal of Transportation Engineering Part A-System, vol. 143, no. 7, 2017.

[15] L. Xu and W. Zhai, "A new model for temporal-spatial stochastic analysis of vehicle-track coupled systems," Vehicle System Dynamics, vol. 55, no. 3, pp. 427-448, 2017.

[16] L. D. A. Corrêa, J. C. Quezada, R. Cottereau, S. C. Aguiar, and C. Voivret, "Randomly-fluctuating heterogeneous continuum model of a ballasted railway track," Computational Mechanics, vol. 60 , pp. 845-861, 2017.

[17] F. T. K. Au, R. J. Jiang, and Y. K. Cheung, "Parameter identification of vehicles moving on continuous bridges," Journal of Sound and Vibration, vol. 269, no. 1-2, pp. 91-111, 2004.

[18] L. Deng and C. S. Cai, "Identification of parameters of vehicles moving on bridges," Engineering Structures, vol. 31, no. 10, pp. 2474-2485, 2009.

[19] P. Asnachinda, T. Pinkaew, and J. A. Laman, "Multiple vehicle axle load identification from continuous bridge bending moment response," Engineering Structures, vol. 30, no. 10, pp. 2800-2817, 2008.

[20] A. Onat, P. Voltr, and M. Lata, "A new friction condition identification approach for wheel-rail interface," International Journal of Rail Transportation, vol. 5, no. 3, pp. 127-144, 2017.

[21] J. Xiang and D. He, "Analysis model of vertical vibration of high-speed train and B(o)gle slab track system," Journal of Traffic and Transportation Engineering, vol. 7, no. 3, pp. 1-5, 2017.

[22] L. Xu and W. Zhai, "Probabilistic assessment of railway vehicle-curved track systems considering track random irregularities," Vehicle System Dynamics, vol. 56, no. 10, 2018.

[23] L. Xu, Z. Li, W. Bai, L. Pan, and Z. Yu, "Numerical simulation platform for slab track systems subjected to a moving vehicle," Advances in Engineering Software, vol. 154, p. 102984, 2021.

[24] J. J. Kalker, "A fast algorithm for the simplified theory of rolling contact," Vehicle System Dynamics, vol. 11, no. 1, pp. 1-13, 1982. 